\title{
THE HYBRID COORDINATE MEASUREMENT SYSTEM AS A RESPONSE TO INDUSTRIAL REQUIREMENTS
}

\author{
Jerzy Sładek ${ }^{1)}$, Robert Sitnik ${ }^{2)}$, Magdalena Kupiec ${ }^{3)}$, Paweł Błaszczyk ${ }^{2)}$ \\ 1) Cracow University of Technology, Laboratory of Coordinate Metrology, Jana Pawta II 37, 31-864 Cracow, Poland \\ (sladek@mech.pk.edu.pl) \\ 2) Warsaw University of Technology, Institute of Micromechanics and Photonics, Św. A. Boboli 8, 02-505 Warsaw, Poland \\ (r.sitnik@mchtr.pw.edu.pl, p.blaszczyk@mchtr.pw.edu.pl) \\ 3) State Higher Vocational School, Institute of Engineering, Staszica 1,33-300 Nowy Sacz, Poland ( $₫$ magdalena.kupiec@gmail.com, \\ +48506 999 479)
}

\begin{abstract}
The tendencies of modern industry are to increase the quality of manufactured products, simultaneously decreasing production time and cost. The hybrid system combines advantages of the high accuracy of contact CMM and the high measurement speed of non-contact structured light optical techniques. The article describes elements of a developed system together with the steps of the measurement process of the hybrid system, with emphasis on segmentation algorithms. Additionally, accuracy determination of such a system realized with the help of a specially designed ball-plate measurement standard is presented.
\end{abstract}

Keywords: optical scanner, CMM, accuracy, segmentation, calibration.

(C) 2010 Polish Academy of Sciences. All rights reserved

\section{Introduction}

Functional, ergonomic and aesthetical parameters are under constant optimization, thus forcing the objects to be of more complex shape. The geometry of the object has to be described accurately by thousands or millions of measurement points. The production process, especially in the aviation, automotive and power industries, requires more effective measurement techniques. Many companies' goal is to measure $100 \%$ of their manufactured products to keep up with the latest production standards. The systems used recently have insufficient means to accomplish this. The new methods should introduce faster measurement speed with high accuracy.

To be compliant with this requirement, several optical sensors have been developed, such as laser triangulation sensors and structured light sensors [1-4]. To speed up measurements all of these sensors have been mounted on CNC's or CMM's heads [5-7]. However, until now, no efficient system has been designed which would consistently integrate optical and contact measurements with automatic data processing in a single measurement.

For this reason a contact-optical measuring system has been proposed and developed [8-10]. It uses the fast optical structured light method for sensors positioned around the working volume. During data analysis a set of points is calculated for a CMM contact head to perform a re-measurement of object areas that have not been registered or require higher accuracy. Both set-ups work in a common coordinate system created according to existing metrological strategies. When these two measurements are completed, a virtual 3D characterization of object is created. Final metrological analysis is performed in classical, certified metrological software. 
The accuracy of the integrated set-up must be verified. Despite manifold works conducted connected with optical systems, issues related to the evaluation of their accuracy still constitute an open problem [11-13]. This fact is mainly connected with a wide variety of structures of optical systems and applied methods of data collection.

A majority of standards for calibration ${ }^{1}$ and verification of optical systems are twodimensional standards [14-16] or spatial patterns whose characteristics may not be specified in terms of contact $[12,17]$. It is known that an important feature of the calibration standard is its similarity to the measured object both in the aspect of shape and optical properties of the surface. Thus, a universal, spatial standard has been developed with elements of a constant curve enabling one to check any freely chosen optical system irrespective of its structure. This standard is used for the calibration of the optical system, as well as for establishing a common optical and CMM coordinate set. Moreover, a procedure for accuracy evaluation of a contactoptical system with the use of such standard has been developed which may be applied in other increasingly popular hybrid systems.

\section{The hybrid system components}

The laboratory hybrid system (see Fig. 1.) is composed of:

- Co-ordinate measuring machine (CMM); a precision, bridge type machine with a movable table. It is equipped with an active probe (probe stylus length $80 \mathrm{~mm}$, tip diameter $8 \mathrm{~mm}$, measurement force $\mathrm{F}=0,5 \mathrm{~N}$, velocity $\mathrm{v}=1 \mathrm{~mm} / \mathrm{sec}$ ) and a thermal correction system. The maximum permissible probing error of CMM, MPEp $=0.8+\mathrm{L} / 400 \mu \mathrm{m}$. The machine is placed in an air-conditioned room.

- Optical scanner(s); the system(s) is based on the structured light measurement technique with digital sinusoidal fringe and Gray code projection and consists of a digital projector and a detector CCD camera. It realizes absolute 3D measurements. The measurement process is performed in two steps: phase measurement (based on an analysis of deformed fringe images) and calibration of the phase values in each pixel of the camera to real $(x, y$, z) Cartesian coordinates [18]. Its calibrated volume is $0.5 \times 0.5 \times 0.3 \mathrm{~m}^{3}$. Depending on the measured part's shape complexity and the required dimensional description one or more directional scanners have to be used.

- Software Mesh 3D for complex system attendance; for the acquisition of 3D "clouds-ofpoints" (consisting of millions of points representing the object) and data pre-processing in the segmentation algorithm. Segmentation algorithm; divides the acquired "cloud-ofpoints" into sets of points belonging to surfaces of defined shape (planes, spheres, cones, cylinders and free form surfaces) which can be compared with their corresponding surfaces of the CAD model.

- Software for the CMM; PC-DMIS 3.5 [19] belonging to the group of CAD/CAE/CAM programs, an advanced package of geometric measurement, graphics oriented, userfriendly and high-performance tools for representing results. Calculation algorithms certified by the PTB (Physikalisch-Technische Bundesanstalt).

- PC Workstation.

- Ball-plate standard (see Fig. 2a) for optical scanner calibration; the developed standard is made of a ceramic glass plate with 25 spheres attached to it. Owing to their spatial character, the spherical shape is best suited for the calibration of such standards in all 3 axes. The thermal expansion coefficient of the plate is very low $\left(\alpha=(0 \pm 0,5) \cdot 10^{-6} 1 / \mathrm{K}\right)$. The spheres are made of chrome plated steel. Their diameter is $\varnothing=25 \mathrm{~mm}$ with a form

\footnotetext{
${ }^{1}$ In this context, calibration is understood as determination of the relation between the coordinates of the detector of the optical system and the real coordinates $(x, y, z)$.
} 
deviation no greater than $\mathrm{R}=1 \mu \mathrm{m}$. The balls of the standard are covered by an antireflection coating. On the basis of a known 3D-standard analysis (the coordinates of the spheres' centres) the parameters of the calibration matrix for the optical scanner are estimated. Next, the model of the standard is measured by an optical scanner. This process takes place many times, with the standard situated in different positions perpendicularly to the scanning axis in the entire measurement volume (see Fig. 2b) with the use of the movable table of the $\mathrm{CMM}^{2}$. This way the coordinates $x, y, z$ are appointed for the measurement volume [18]. The application of the spherical standard in the calibration of the optical system enabled the calibration of the optical system in conditions of real measurement and thus ensured traceability of the measurement.

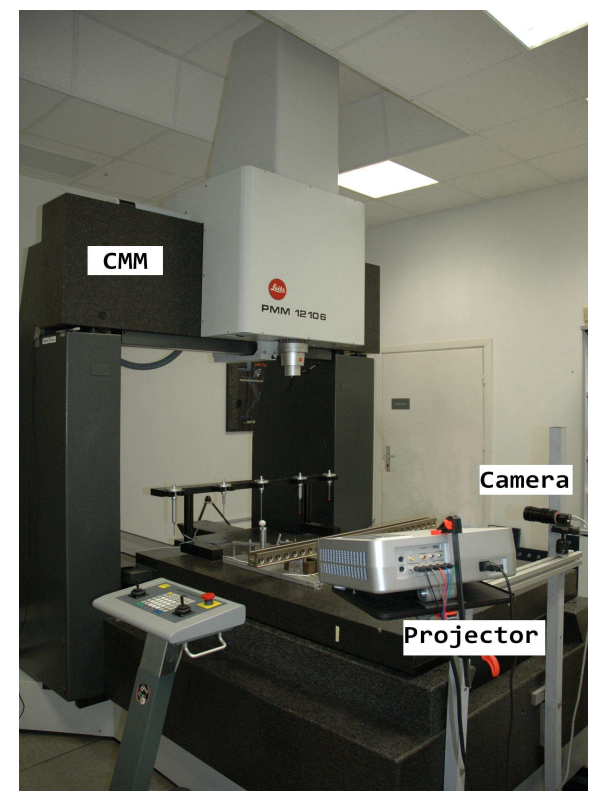

Fig. 1. Laboratory hybrid system.

a)

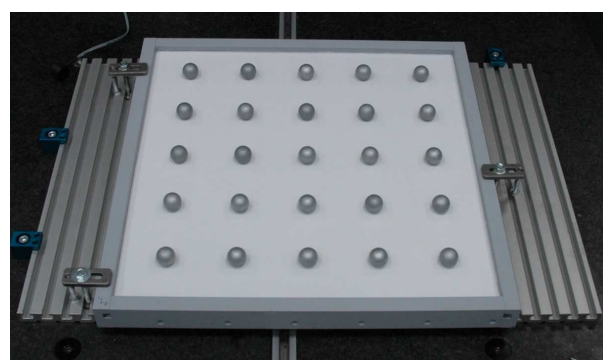

Fig. 2. a) Ball-plate standard; b) Calibration process of optical system. b)

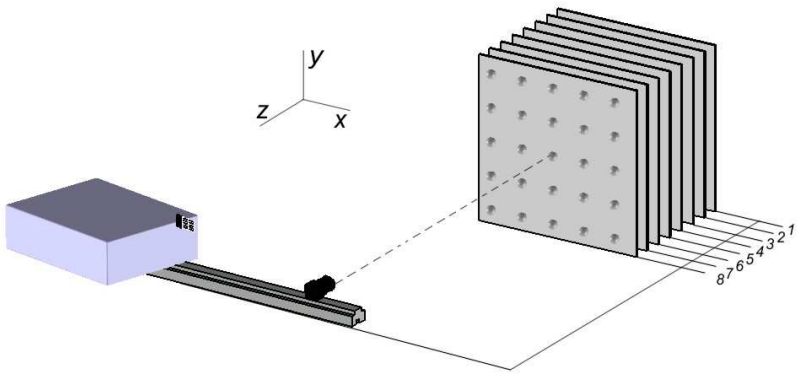

\section{Measurement process of the hybrid system}

The hybrid system consists of subsequent steps (see Fig. 3):

- The unified coordinate system is created with the use of the CMM and a developed calibration ball-plate according to existing metrological strategies.

\footnotetext{
${ }^{2}$ There is the option without movable table of the CMM with the use of a linear table and measurement of calibration model coordinates in each of table positions.
} 
- The next step is a transformation of relative coordinate systems (from optical measurement) to absolute ones created in the first step (carried out by the ball-plate standard measurement and the calculation of an optical-to-model transformation).

- The examined object is measured by a fast optical system(s). As a result we obtain a 3D "clouds of points".

- The cloud of points is imported into the segmentation algorithm. Points belonging to the areas uniform shape are grouped together. The algorithm attempts to recognize as being of known shape such as planes, spheres, cylinder. All of the groups which we are not able to univocally assign to a specific surface are marked as free form.

- Localization of discontinued areas and areas with holes takes place. These areas are pointed out to be re-measured by the CMM.

- When these two measurements are completed, a final metrological analysis is performed using a classic, certified metrological software PC- DMIS.

To obtain the information about the shape of the entire object, it has to be measured from several directions with the optical system. The "cloud-of-point" data from each directional measurement have to be put together. The resultant data set holds the information about the entire object. There is no distinction between individual surfaces. Segmentation is necessary so that only the points belonging to each surface are compared with their corresponding surfaces of the CAD model. The program emulates a CMM when exporting the measurement data to the PC-DMIS program sending each group of points separately for metrological evaluation of the given surface. Certified metrological software is indispensible because it is acknowledged by industry for the evaluation of measurement results of manufactured products.

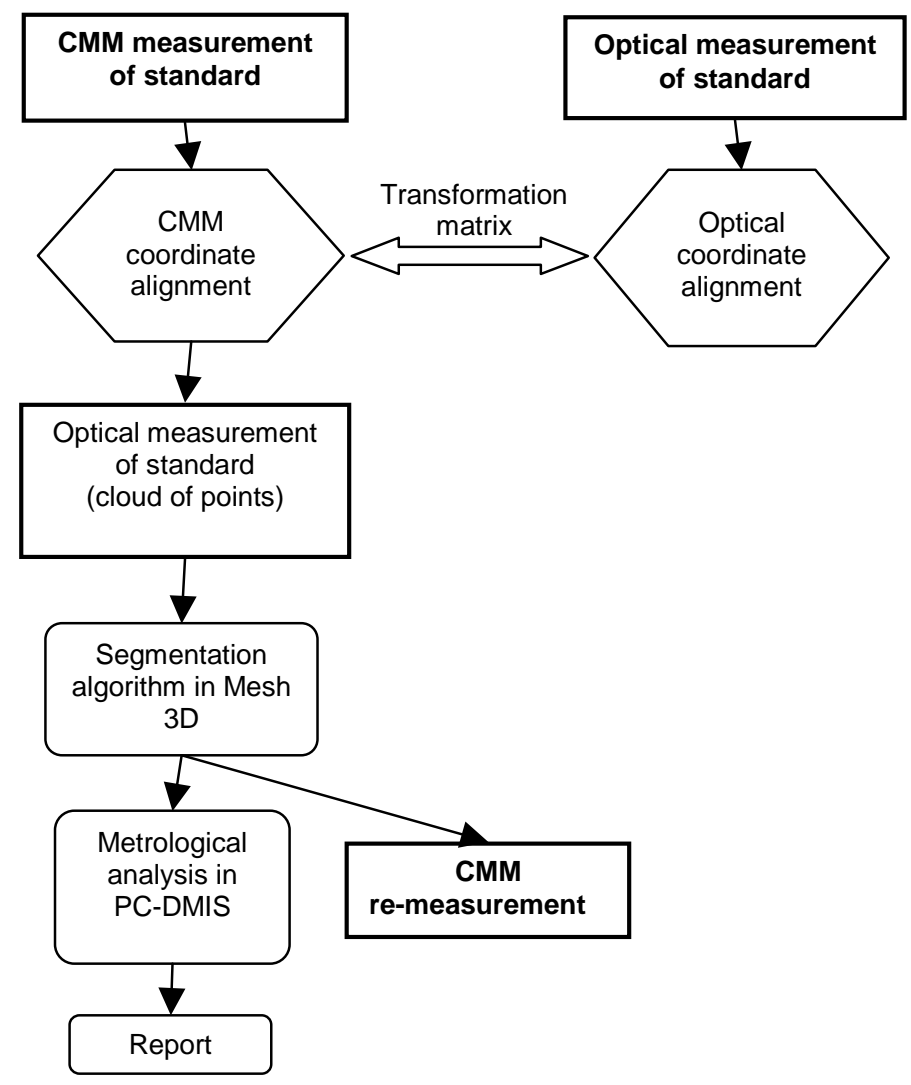

Fig. 3. Scheme of hybrid measurement process. 


\subsection{Segmentation process}

There have been several approaches to 3D measurement data segmentation [20-22]. The majority of the segmentation algorithms intended to be used in reverse engineering or digital shape reconstruction utilize mesh data. Data intended for metrological evaluation should be in the form of a cloud-of-points which is unprocessed, straight from the scanning process. Cloud-of-point data also contains a considerably greater amount of measurement points than mesh data.

Various segmentation algorithms require different amounts of user attention. It would be most advantageous to exclude the user from the segmentation process. However at this point it is impossible. In our segmentation method the user is required only for the final segmentation results assessment.

The segmentation process is a recognition problem where we attempt to find higher-level patterns from given low-level information. The whole process involves dividing the cloud-ofpoint data into areas of defined surface shape (planes, spheres, cones, cylinders and free form surfaces).

Different data sets will have different numbers of points, densities, sizes and noise. Due to the fact that the cloud-of points is assumed to be unsorted, point adjacency has to be determined. Points which lie close to each other in space may be located in different places of the data set. Point adjacency is determined by calculating the distance between the points in space and choosing the closest ones. Local geometrical shape features are calculated based on the relative position of these closest points.

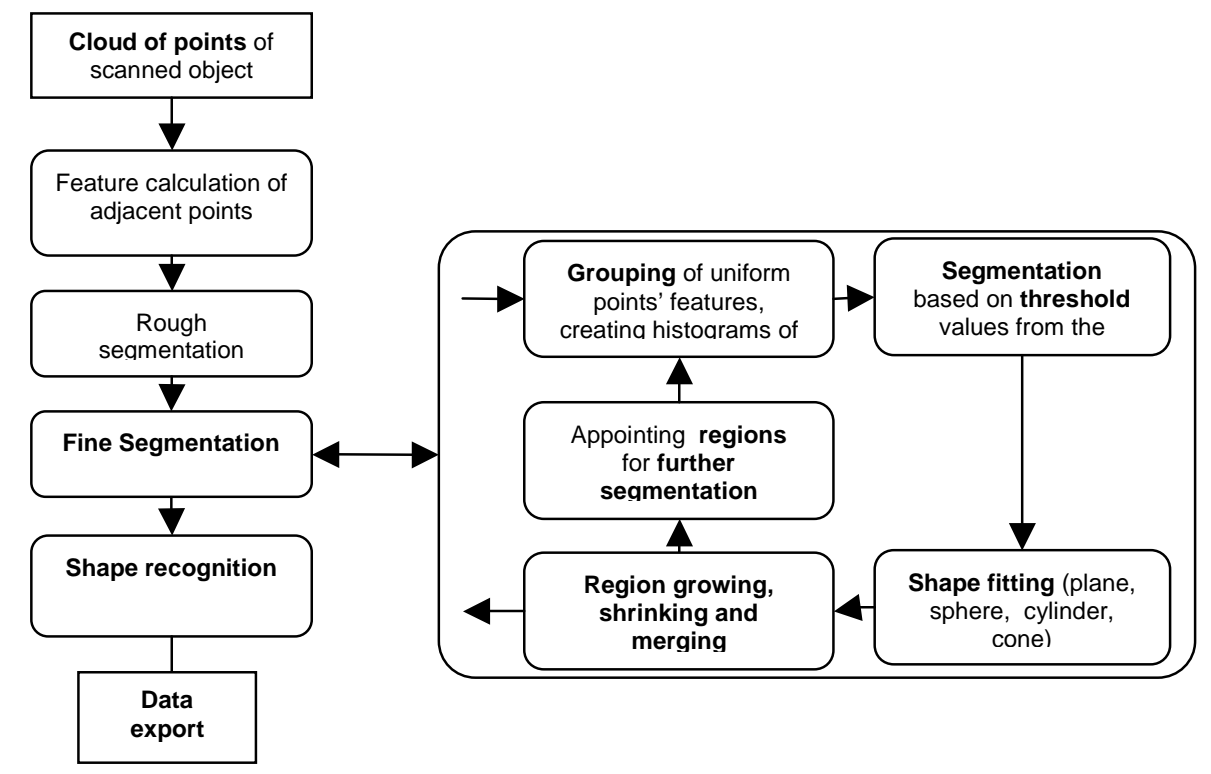

Fig. 4. Scheme of the segmentation process.

To reduce the amount of points being processed at a time, thus improving cloud segmentation speed, rough segmentation is used. The calculated feature values are used to determine points near discontinuities such as edges (Fig. 5a.). The appointed points separate the cloud into initial regions. At this point way to distinguish between edge points and noisy points.

Fine segmentation is a multi-pass, iterative process that further divides the initial cloud regions. At first a histogram of feature values is created for each initial region. By means of a histogram analysis we are able to determine if the region has been properly segmented. Further analysis of the histograms of the correctly segmented regions enables us to determine 
their most probable shape. Since shape fitting is a time-consuming process it would be advantageous to fit only the proper shape. To evaluate shape fitting correctness the RMS value of the distance of all the points in the region from the fit shape is calculated and compared with the average noise in the cloud. A greater value indicates an incorrect fit. This either means that the region includes erroneous points and is intended for region shrinking (elimination of non fitting points) or is of a different shape (the next most probable shape is intended to be fit). If no shape can be fit the region is assigned for further segmentation and the entire process is repeated for such a region. The process is also repeated for regions that have not been correctly segmented during rough segmentation (rejected during histogram analysis). If shape fitting was a success the adjacent points of the region are checked if they could not be joined with the region. Due to measurement noise, regions that should be treated as a single region are separated from each other. The algorithm attempts to merge adjacent regions whose surfaces that have been fit have similar parameters. If the fitting result for the two regions put together is no worse than the individual fitting results, the regions are merged.

As the result of the segmentation process we receive groups of points comprised of areas of uniform shape. The shape of each region is recognised (Fig. 5b.) as being one of the following: plane, sphere, cone, cylinder or unknown (free form). This data can be exported for metrological software.

a)

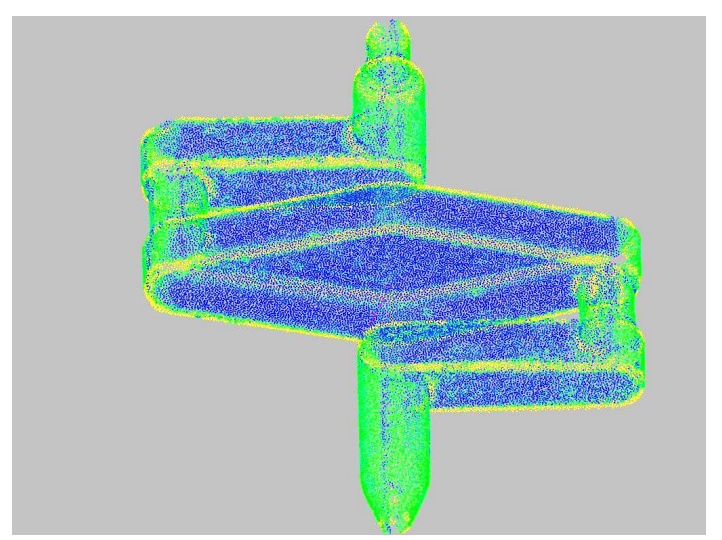

b)

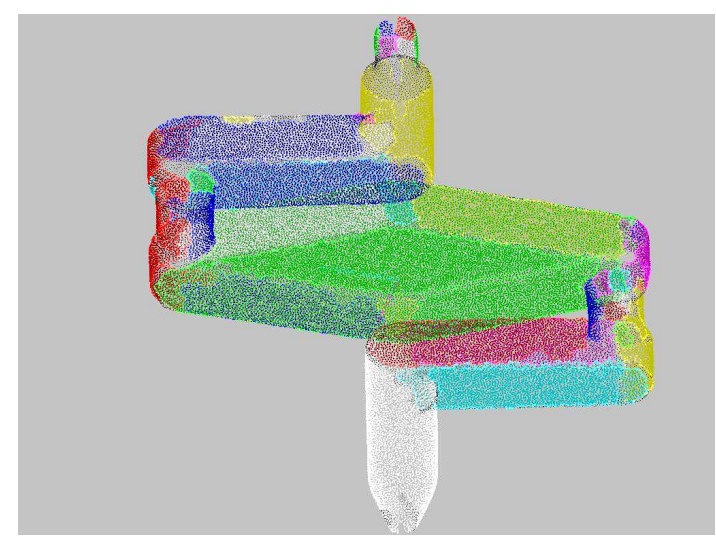

Fig. 5. Segmentation results: a) feature value distribution in cloud (blue-lowest, yellow-highest); b) segmented cloud (each color represents a different region).

\section{Indication error $\mathbf{E}$ of the hybrid system}

After building the hybrid system we had to verify its accuracy. It is assumed that if the hybrid system is used for geometrical measurement, it should be verified like other devices for coordinate measurements. Therefore, it has to be calibrated and verified basing on the procedures for inspection of length measurement (ISO 10360 - 2 norm [23] and recommendation of VDI/VDE 2617 - 6 [24]). The procedures compare measurement values with the known calibrated values of material standards of size. Thus, one may obtain an indication error for size measurement $\mathrm{E}$ of the measuring system. The value of this error is described by the following formula:

$$
E=l_{m}-l_{s},
$$

where:

- $l_{m}-$ measured length indicated by the measuring system;

- $l_{s}$ - nominal of the measured length.

To verify the accuracy of the hybrid system a similar ball-plate standard was used. The ball-plate standard nominal values (the distance between the centers of the balls) were 
obtained from a reference CMM. In this way the unbroken chain of comparisons (calibration chain) is ensured and measurement traceability to the SI-unit "meter" is kept.

The ball-plate standard has been measured in eight different positions (as shown in Fig. 6), three times in each position, in a bi-directional manner. Positions 1, 2, 3, 4 are perpendicular to the optical axis of the camera (in plane $x y$ of the hybrid system), positions 5, 6, 7, 8 are in space $x y z$ of the hybrid system ${ }^{3}$.
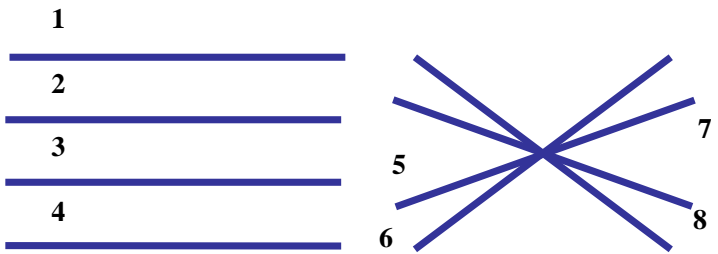

Fig. 6. Positions of the ball-plate standard in the work area of the scanner - top view.

The common coordinate system was created each time using a three-boundary sphere as its base. Next, coordinate systems of optical scanner and CMM were transformed into a common one. The measurement result of the optical scanner is rendered in the form of a 3D "cloud of points". The points belonging to the spheres were isolated from the "cloud of points" into the segmentation algorithm and transferred to the PC - DMIS software in dms format. However, some areas were pointed out to be re-measured by the CMM.

In metrological software the data from the optical system and CMM were replaced by substitute elements. Next, the coordinates of the centers of the spheres were determined. After the calculation of the distances between them, it was possible to compare them with their nominal values. As a result, we obtained deviations of the distances from nominal values. These are known as the indication error E.

a)

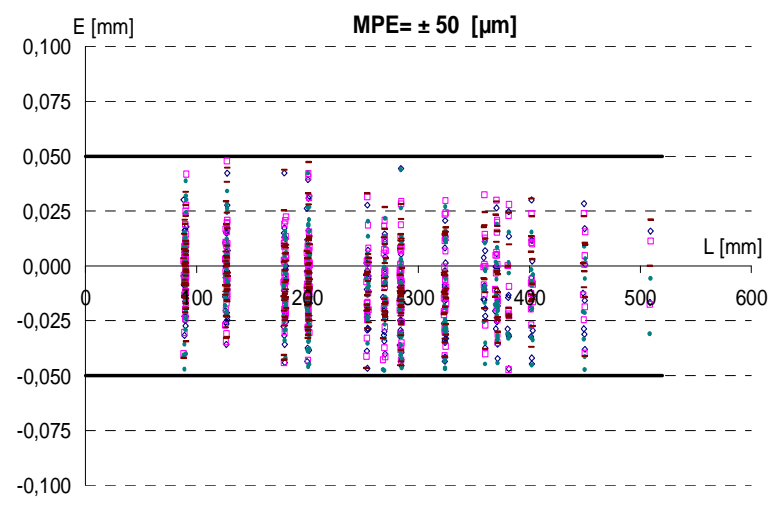

b)

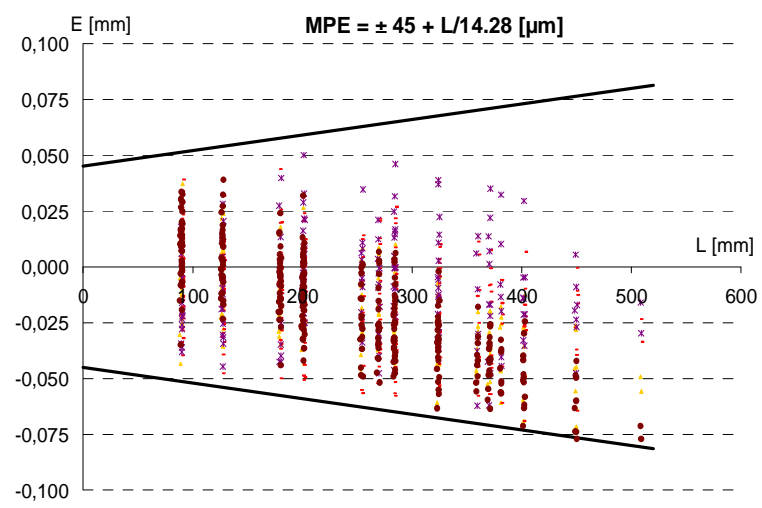

Fig. 7. The area of MPE (EL) of the hybrid system a) in $x, y$ plane; b) in $x, y, z$ space.

Fig. 7a shows values of the indication error $\mathrm{E}$ for the ball-plate standard positions in $x, y$ plane of the hybrid system. The formula of the maximum permissible error of length measurement $\mathrm{MPE}(\mathrm{EL})$ can be written as: $\mathrm{MPE}(\mathrm{EL})= \pm 50 \mu \mathrm{m}$ for this position. However, for the remaining positions (see Fig. 7b) MPE (EL) $= \pm 45+\mathrm{L} / 14,28 \mu \mathrm{m}$. Hence, it indicates that along with an increase of the angle at which the standard is curved towards the scanner, indication errors multiply and behave linearly. This is connected with the optical system calibration procedure, where values of $x, y$ are designated on the basis of the coordinates of

\footnotetext{
${ }^{3}$ The standard must be in the field of view of the camera and projector (the angular position should cover values within the range $<-45^{\circ}, 45^{\circ}>$ ).
} 
the centers of the spheres in the standard and the values $z$ on the basis of a shift of this standard in the measuring volume of the hybrid system, which is the cause of additional errors.

\section{Summary}

We presented a hybrid system that integrates contact and no-contact measurement methods. It responds to the need of modern industry requirements mainly in the task of speeding up of the measurement process.

The measurement process starts from fast optical acquisition by a structured light system and then, in the segmentation algorithm, optical measurement points belonging to areas of uniform shape are grouped together. We showed the results of the use of the segmentation algorithm. The grouped data are exported for metrological software. Moreover, the localization of discontinued areas and areas with holes takes place in order to be re-measured by the CMM.

Accuracy determination (consistent with the idea of the coordinate-based measurement technique) is realized with the help of a ball-plate measurement standard. Its metrological characteristic is given based on its measurement on a reference, high accuracy CMM. In this way measurement traceability is ensured and associated with national and international standards of units of length.

The obtained results presented in this paper meet the standards of the automotive industry and prove that the hybrid concept is promising, therefore, a continuation of its development is justified.

\section{Acknowledgment}

This work was performed under the grant No. R17 00102 financed by the Polish Ministry of Science and Higher Education.

\section{References}

[1] Wang, G., Zheng, B., Xin, L., Houkes, Z. (2002). Modeling and calibration of the laser beam scanning triangulation measurement system. Robotic and Anatonomous Systems, 40, 267-277.

[2] Zhang, G., Wei, Z. (2002). A novel calibration approach to 3D vision inspection". Optics \& Laser Technology, 34(5), 373-380.

[3] Zhang, J., Djordjevich, A. (1999). Study on laser stripe sensor. Sensors and Actuators, 72(3), 224-228.

[4] Xie, Z., Wang, J., Zhang, Q. (2005). Complete 3D measurement in reverse engineering using a multi-probe system. Machine Tools \& Manufacture, 45, 1474-1486.

[5] Xinmin, L., Zhongqin, L., Tian, H., Ziping, Z. (2001). A study of a reverse engineering system based on vision sensor for free-form surfaces. Computer \& Industrial Engineering, 40, 215-227.

[6] Son, S., Park, H., Lee, K.H. (2002). Automated laser scanning system for reverse engineering and inspection. International Journal of Machine Tools \& Manufacture, 42, 889-897.

[7] Chang, M., Lin, K.H. (1999). Non-contact scanning measurement utilizing a space mapping method. Optics and Lasers in Enginnering, 30, 503-512.

[8] Sitnik, R., Sładek, J., Kupiec, M., Błaszczyk, P., Kujawińska, M. (2006). New concept of fast hybrid contact and no-contact measurement for automotive industry. Conf. Photonics Europe Strasbourg. France, 619803.

[9] Sładek, J., Sitnik, R., Kupiec, M., Błaszczyk, P. (2006). The new hybrid method for fast and precision measurement. In Proceedings of XVIII IMEKO World Congress Metrology for a Sustainable Development, Rio de Janeiro, Brazil, 134-138. 
[10] Kupiec, M. (2007). Optical - Contact Coordinate Measurement Method. Ph.D. dissertation. Cracow University of Technology.

[11] Gonnet, J.P., Isheil, A., Fontaine, J.F. (2007). Parameters analysis influencing 3D measurement without contact by laser scanner to establish a local correction procedure. In Proceedings of 10th CIRP Conference on Computer Aided Tolerancing. Erlangen, Germany.

[12] Marshall, S.J., Whiteford, D.N., Rixon, R.C. (2001). Assessing the performance of 3d whole body imaging systems. Paris, France.

[13] Weckenmann, A., Weickmann, J. (2006). Optical inspection of formed sheet metal parts applying fringe projection systems and virtual fixation. Metrol. Meas. Syst., 13(4), 321-334.

[14]El-Hakim, S.F., Beraldin, J.A., Blais, F. (1995). A Comparative Evaluation of the Performance of Passive and Active 3-D Vision Systems. Proceedings of St. Petersburg Conference on Digital Photogrammetry. St. Petersburg, Russia, NRC 39160.

[15] Gühring, J., Brenner, C., Böhm, J., Fritsch, D. (2000). Data processing and calibration of a cross-pattern stripe projector. In Proceedings of ISPRS Congress 2000. Amsterdam, Netherlands, IAPRS 33(5).

[16] Informational material GOM' company. http://www.gom.com

[17] Informational material Cognitens' company. http://www.cognitens.com

[18] Sitnik, R. (2002). A fully automatic 3D shape measurement system with data export for engineering and multimedia systems. Ph.D. dissertation. Warsaw University of Technology.

[19] Informational material Wilcox Associates’ Inc. http://www.pcdmis.com

[20] Vieira, M., Shimada, K. (2005). Surface Mesh Segmentation and Smooth Surface Extraction through Region Growing. Computer Aided Geometric Design, 22(8),771-792.

[21] V'arady, T., Facello, M.A., Ter'ek, Z. (2007). Automatic Extraction of Surface Structures in Digital Shape Recon-struction. Computer-Aided Design, 39(5), 379-388.

[22] Heckbert, P.S., Garland, M. (1997). Survey of Polygonal Surface Simplification Algorithms. Multiresolution Surface Modeling Course. SIGGRAPH'97.

[23] ISO 10360-2. (2001) Geometrical Product Specifications (GPS), Acceptance and reverification tests for coordinate measuring machines (CMM) - Part 2: CMMs used for measuring size Standard.

[24] VDI/VDE 2617-6. (1997). Genauigkeit von Koordinatenmessgeräten - Kenngrössen und deren Prüfung Koordinatenmessgeräte mit optischer Antastung - Grundlagen. 